\title{
Structural basis for termination of AIM2-mediated signaling by p202
}

\author{
Cell Research (2013) 23:855-858. doi:10.1038/cr.2013.52; published online 9 April 2013
}

\section{Dear Editor,}

Sighting and binding of double-stranded DNA (dsDNA) by a sensor in the cytoplasm trigger the activation of the immune-surveillance pathways [1]. The crystal structure of absent in melanoma 2 (AIM2) bound with DNA conclusively defines the role of AIM2 as a sensor in the innate immune system [2]. AIM2 belongs to the PYHIN family of proteins and contains a pyrin domain (PYD) followed by a hematopoietic interferon-inducible nuclear protein (HIN) domain (Figure 1A). AIM2 binds DNA via the HIN domain and recruits the adaptor protein apoptosis-associated speck-like protein containing a caspase recruitment domain (ASC) via the PYD. ASC in turn recruits caspase-1 via CARD-CARD interaction, resulting in the formation of inflammasomes comprised of AIM2, ASC and caspase-1. The molecular crowding of the AIM2 inflammasome ensures the proteolysis and transactivation of caspase-1. Activated caspase- 1 cleaves pro-IL-1 $\beta$ and pro-IL-18 into their mature proinflammatory forms $[3,4]$.

The termination of inflammatory responses originated from inflammasomes can be accomplished by employing naturally occurring dominant-negative antagonists [4]. Dominant-negative proteins are similar to their canonical counterparts except for a missing effector domain, so that they cannot relay the signals any further. They outcompete their canonical counterparts for ligands or binding sites and thus block the downstream signal transduction. Such regulation is essential for maintaining cellular homeostasis. To regulate inflammasome activation, mice have evolved a strategy that has so far not been discovered in humans. Mice use the HIN-only protein, p202, to sequester cytoplasmic dsDNA and render it unavailable for its canonical sensor, AIM2 [4]. p202 contains two $\mathrm{HIN}$ domains (HINa and HINb), but lacks the PYD (Figure 1A). Therefore, p202 is unable to recruit the adaptor $\mathrm{ASC}$, and its binding to DNA results in the termination of inflammasome signaling. The significance of p202 in the regulation of the innate immune responses is exemplified by the fact that dysregulation of $\mathrm{p} 202$ function has been linked to increased susceptibility to systemic lupus erythematosus [5].

To more clearly understand the mechanism of inhibition of AIM2-mediated signaling by p202, it is essential to solve the structure of p202 in complex with DNA and compare it with that of AIM2 complexed with DNA. p202 has so far only been detected in mice. To compare the structure of AIM2 and p202 from the same species, we first solved the structure of the HIN domain of murine AIM2 (mAIM2) in complex with dsDNA to 2.23 $\AA$ resolution (Supplementary information, Table S1). Although a 12-base pair (bp) long dsDNA was used for the crystallization, the HIN domain of mAIM2 seems to have lined up the DNA oligonucleotides end to end, generating an appearance of a long and contiguous stretch of B-form DNA with putative major and minor grooves. As expected, the overall structure of the HIN domain of mAIM2 (Figure 1B) closely mirrors the structure of its human counterpart [2] (Supplementary information, Figure S1). Minor deviations are observed at the N-terminus and in the loop regions. The surface electrostatic potential distribution is similar, implying that the mechanism of tethering dsDNA is similar between human and mouse AIM2.

The HIN domain of AIM2 consists of two oligonucleotide/oligosaccharide (OB) folds [6] linked via a flexible linker (Figure 1B). The proximal and distal OB folds are referred to as OB1 and OB2, respectively. Similar to the human ortholog, mAIM2 uses the helix-loop-helix motif located in the linker to engage DNA (Figure 1C). Specifically, a short helix containing two turns is inserted horizontally to the vertical axis of the DNA spiral (Figure 1C). Amino acids from the loop connecting helices $\alpha 1$ and $\alpha 2$, and from helix $\alpha 2$ interact with the major groove (Figure 1C). A couple of interactions between OB2 and the DNA backbone are also observed. Residues N244, N245, K248, R249, R251, R255, K258, Q262, K273, K291, K313 and R315 provide a contiguous stretch of positive potential for binding the negatively-charged DNA backbone (Figure 1D and Supplementary information, Figure S2). Except for the side chain of K248 
of mAIM2 donating two hydrogen bonds to the DNA bases, all other protein-DNA interactions are limited to the DNA backbone (Supplementary information, Figure S3). These results demonstrate that the HIN domain of mAIM2 primarily uses the linker connecting the two OB folds to bind dsDNA. Sequence alignments of the linker region among AIM2 homologs from different spe- cies reveal that although only T253 and R255 are highly conserved (Supplementary information, Figure S4), the linker regions of AIM2 homologs are predominantly populated with positively-charged amino acids. Therefore, other AIM2 homologs likely also use the positive potential of the linker region to tether DNA.

Only the HINa domain (amino acids 46-246) of the
A
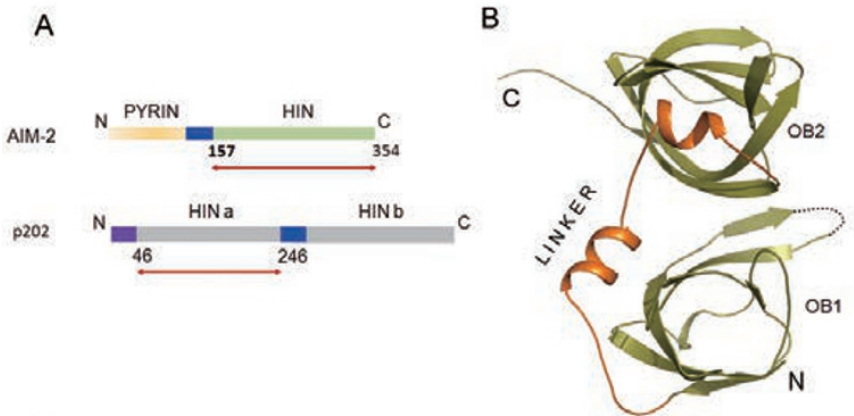

D

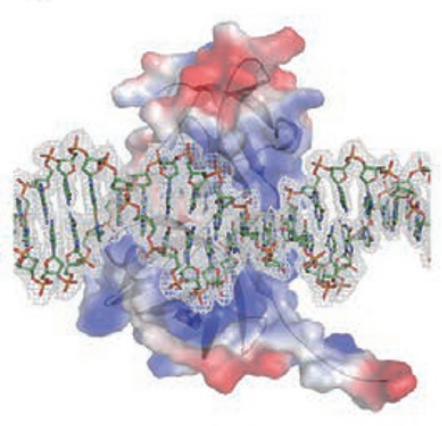

E

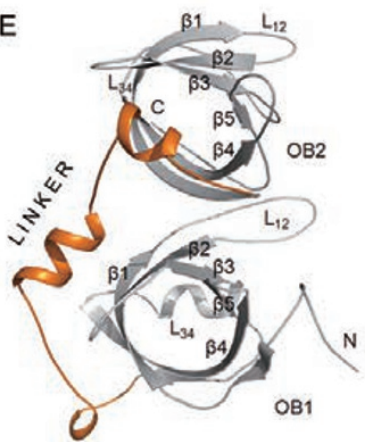

C

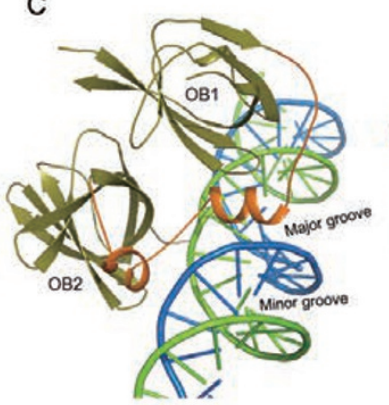

F

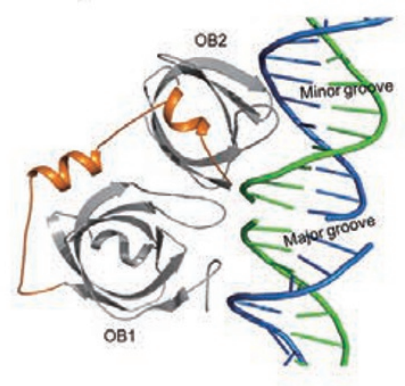

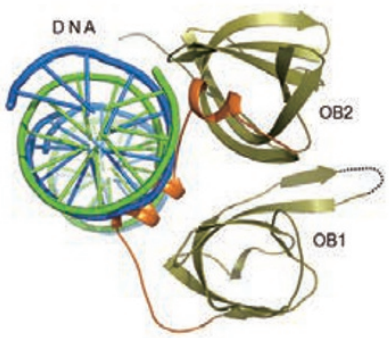

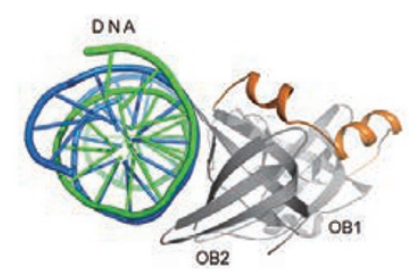

I

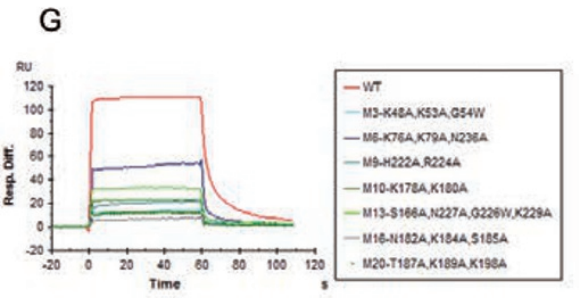

H
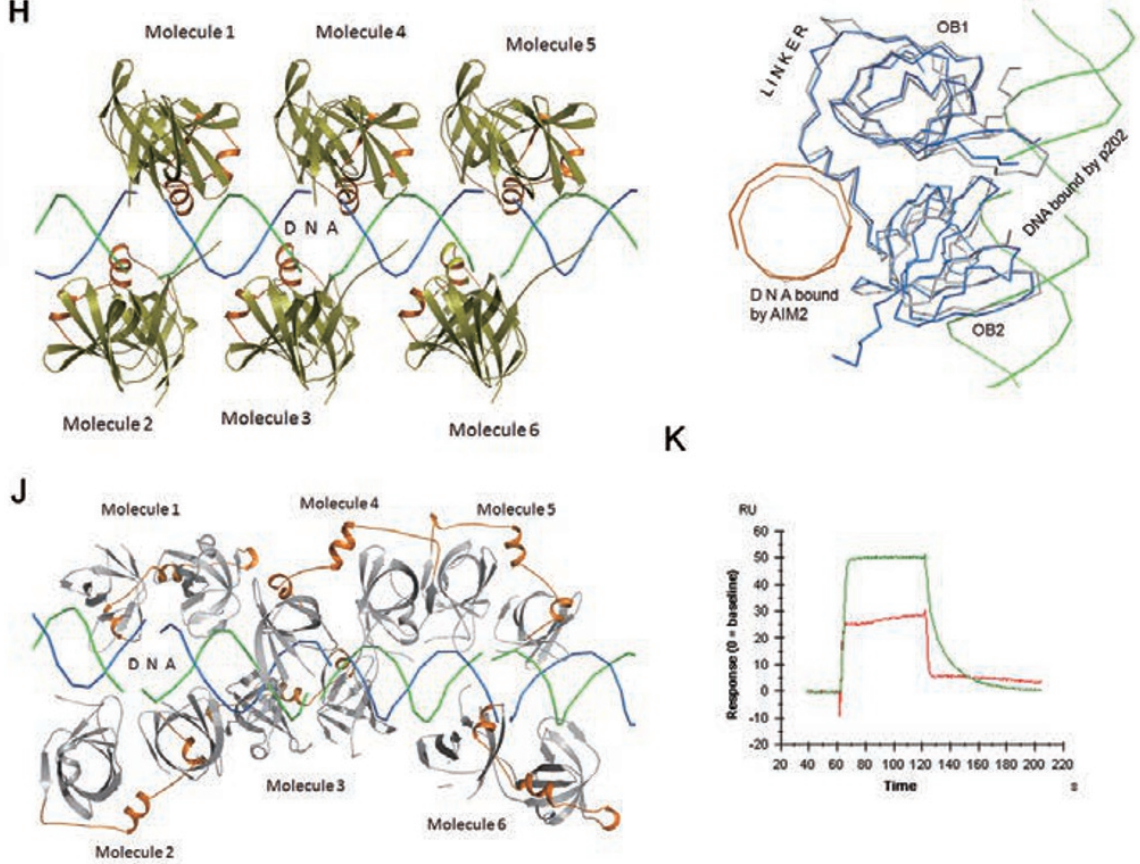

K

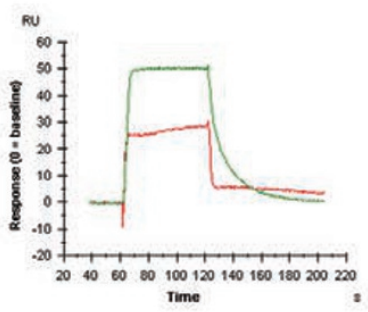


Figure 1 Structural basis for the termination of AIM2-mediated signaling by p202. (A) Schematic diagram of domain arrangement of mAIM2 and p202. Arrows indicate the length of the construct used for structural studies. (B) Cartoon representation of the structure of the HIN domain of mAIM2 bound with DNA. (C) Side view of the cartoon representation of the structure of the HIN domain of mAIM2 bound with dsDNA (left panel). Right panel shows the top view of the structure showing a helix from the linker inserted into the DNA spiral. DNA strand are shown in blue and green. (D) Electrostatic surface potential representation of the HIN domain of mAIM2 bound with dsDNA. 2Fo-Fc electron density map for DNA contoured at $1 \sigma$ is shown. Positive potential is shown in blue, negative potential in red. DNA is shown as sticks. (E) Cartoon representation of the structure of the HINa domain of p202. Secondary structural elements are labeled according to the canonical OB-fold convention. (F) Side view of the structure of p202-HINa bound with dsDNA (left panel). Right panel shows the top view of the same structure showing loops protruding out from the $\beta$-barrel of OB-fold. Strands of DNA are shown in blue and green. (G) Surface plasmon resonance (SPR) assay of the DNA-binding affinity of wild-type p202 HINa domain and its mutants harboring clusters of mutations (M3, M6, M9, M10, M13, M16 and M20) around the DNA-binding region. Data shown represent the binding to AT-rich dsDNA (5'-TTATATATATATATATATAA-3'). (H) Cartoon representation of the mAIM2 HIN molecules bound to dsDNA. (I) Modes of dsDNA binding by the HIN domains of p202 and AIM2. C $\alpha$ atoms of the HINa domain of p202 (grey) bound with DNA (green ribbon) are superimposed over $\mathrm{C} \alpha$ atoms of the HIN domain of murine AIM2 (blue) bound with DNA (orange ribbon). (J) Cartoon of the p202 HINa molecules bound with DNA. (K) The HINa domain of p202 (green) binds more DNA than mAIM2 HIN domain (red) when assayed under identical conditions using equal molar ratios of protein and DNA.

mouse p202 could be expressed in quantities sufficient for structural studies by us. We solved the structure of the p202 HINa domain at $2.89 \AA$ resolution (Supplementary information, Table S1). Similar to the structure of the HIN domain of human AIM2 [2], the p202 HINa domain consists of two OB folds, proximal OB1 and distal OB2. The long linker connecting OB1 and OB2 contains a helix-loop-helix motif (Figure 1E). Each OB fold is made up of a $\beta$-barrel consisting of seven $\beta$-strands. The canonical $\mathrm{L}_{12}$ loop of OB1 is unusually long and protrudes out of the $\beta$-barrel like a hook (Figure $1 \mathrm{E})$. In addition, the canonical strand $\beta 4$ in $\mathrm{OB} 1$ is long and highly twisted, suggesting the existence of a pent-up tensile force. While the $\mathrm{L}_{23}$ loop connecting the $\beta 2$ and $\beta 3$ strands is short, the $L_{34}$ loop of OB1 is relatively long and has a very short helix embedded at the centre. Unlike OB1, the $\mathrm{L}_{12}$ loop of OB2 is short. However, the loop connecting the $\beta 4$ and $\beta 5$ strands is long and is projected out of the $\beta$-barrel. Thus, the p202 HINa domain is made up of two similar OB folds that show some differences in the loop regions.

Next we solved the structure of p202-HINa bound with $14-b p$ dsDNA at $3.0 \AA$ resolution (Supplementary information, Table S1). Comparison of the native structure with the DNA-bound structure reveals that the p202-HINa does not undergo large-scale conformational changes upon DNA binding. The overall topology of the structure closely mirrors the HIN domain of mAIM2 (Supplementary information, Figure S5). The most striking structural difference between the HIN domains of p202 and AIM2 lies in the distribution of the charge on the surface. The positive potential observed in the linker region of AIM2 involved in DNA binding is significantly reduced in the linker of p202-HINa (Supplementary information, Figure S6). Instead, the region around the
$\mathrm{N}$-terminus of OB1 and the convex surface of the sheet formed by strands $\beta 1, \beta 2, \beta 3$ and $\beta 7^{\prime}$ (non-canonical strand) of OB2 show enhanced positive potential. These differences in the distribution of surface electrostatic potential allow p202 to employ a DNA-binding mode that is different from that of AIM2. Consequently, the differences in the DNA-binding mode have implications for the role of p202 as an AIM2 antagonist.

We next studied the molecular details of the DNAbinding mode of p202. Interestingly, just like AIM2, the p202 HINa domain seems to have bridged the DNA molecules end-to-end, giving an appearance of a long continuous stretch of DNA. Unlike AIM2, the linker connecting the OB folds in $\mathrm{p} 202-\mathrm{HINa}$ does not interact with DNA; instead the loops from both OB1 and OB2 contact DNA (Figure 1F). Except for two hydrogen bonds formed between the side chain of K53 and the DNA bases, all other interactions are limited to the DNA backbone (Supplementary information, Figure S7). The details of the interactions are summarized in Supplementary information, Table S4 and Figure S8. To verify the protein:DNA interface observed in the crystal structure, we generated several mutants (M3, M6, M9, M10, M13, M16, M20) containing clusters of mutations of amino acids at the interface and tested their DNA-binding ability using the surface plasmon resonance (SPR) assay. All mutants showed remarkable decrease in affinity for dsDNA (Figure 1G, Supplementary information, Figures S9S13 and Table S2). Therefore, the protein:DNA interface observed in the crystal structure is functionally relevant. All together, the p202 HINa domain primarily uses electrostatic interactions to bind DNA. Interestingly, none of the amino acids involved in DNA binding are conserved among the predicted p202 homologs (Supplementary information, Figure S14), suggesting that these p202 ho- 
mologs probably do not perform the same function.

Crystallographic snapshots of the interactions of the HIN domains of AIM2 and p202 to DNA provide unique perspectives on how AIM2 activates the inflammasome signaling through molecular crowding and how p202 downregulates the signaling (Figure $1 \mathrm{H}$ and $1 \mathrm{~J}$ ). The distance between two adjacent molecules of the mAIM2 HIN domain bound to DNA is less than $10 \AA$. Such a mode of binding forms the platform for the recruitment of ASC and caspase-1, leading to oligomerization of the proteins and activation of the downstream signaling. In contrast, p202 contacts a much larger span of DNA and probes a much wider range of topological niches of DNA. This is accomplished by cleverly switching the structural elements used for binding DNA (Figure 1I). Instead of using the linker to bind DNA as shown for AIM2, p202-HINa adopts the classical approach used by a number of OB-fold-containing proteins for tethering DNA [6] - primarily using the loops protruding out of the canonical $\beta$-barrel. This strategy permits p202 to bind to a larger surface area of dsDNA. Indeed, binding of DNA to the HIN domains of p202 and AIM2 results in a burial of the total accessible surface area of $922 \AA^{2}$ and $477 \AA^{2}$, respectively (Supplementary information, Figure S15). Therefore, the p202 HINa domain binds DNA with a much higher affinity than AIM2 (Figures 1K). Thus, when these two proteins are present in equal amounts and compete for the same dsDNA, p202 likely occupies most of the binding sites on the dsDNA. Even if AIM2 has bound to DNA, p202 could still intercalate by binding to the minor groove and prevent the oligomerization of AIM2 and subsequent activation of caspase-1. Our structural studies provide important insights into the mechanism of downregulation of AIM2-mediated signaling by $\mathrm{p} 202$.

\section{Accession codes}

The atomic coordinates and structure factor files for murine AIM2-HIN in complex with dsDNA, p202-HINa and p202-HINa in complex with dsDNA have been deposited in PDB under the ac- cession codes 4JBJ, 4JBK and 4JBM, respectively.

\section{Acknowledgments}

We thank Prof Katryn J Stacey for generously providing the construct of murine p202-HINa. We are grateful to Y Han, YY Chen and Y Wang at the Protein Science Core Facility of IBP for technical help with X-Ray diffraction, ITC experiments and automatic crystallizations, respectively. This work was supported by grants from the Ministry of Health of China (2013ZX10004-602), the Ministry of Science and Technology of China (2013CB911103, 2009CB918803, 2011CB911103) and the National Natural Science Foundation of China $(31270795,31200559,31070660$, 31021062).

Heng $\mathrm{Ru}^{1, *}$, Xiangmin $\mathrm{Ni}^{1,{ }^{*}}$, Lixia Zhao ${ }^{1,3, *}$, Christopher Crowley ${ }^{2}$, Wei Ding ${ }^{1}$, Li-Wei Hung ${ }^{4}$, Neil Shaw ${ }^{1,3}$, Genhong Cheng ${ }^{2}$, Zhi-Jie Liu ${ }^{1,3}$

${ }^{I}$ National Laboratory of Biomacromolecules, Institute of Biophysics, Chinese Academy of Sciences, Beijing 100101, China, ${ }^{2}$ Department of Microbiology, Immunology and Molecular Genetics, University of California Los Angeles, Los Angeles, CA 90095, USA; ${ }^{3}$ Institute of Molecular and Clinical Medicine, Kunming Medical University, Kunming 650500, China: ${ }^{4}$ Physics

Division, Los Alamos National Laboratory, Los Alamos, NM 87545, USA

"These three authors contributed equally to this work.

Correspondence: Zhi-Jie Liu ${ }^{\mathrm{a}}$, Genhong Cheng ${ }^{\mathrm{b}}$, Neil Shaw ${ }^{\mathrm{c}}$

${ }^{\mathrm{a}}$ Tel: +86-10-64857988; Fax: +86-10-64888426

E-mail: zjliu@ibp.ac.cn

${ }^{\mathrm{b}}$ Tel: +1-310-825-8896; Fax: +1-310-206-5553

E-mail: gcheng@mednet.ucla.edu

'Tel: +86-10-64888252; Fax: +86-10-64888426

E-mail: neilshaw@moon.ibp.ac.cn

\section{References}

1 Hornung V, Latz E. Nat Rev Immunol 2010; 10:123-130.

2 Jin T, Perry A, Jiang J, et al. Immunity 2012; 36:561-571.

3 Hornung V, Ablasser A, Charrel-Dennis M, et al. Nature 2009; 458:514-518.

4 Roberts TL, Idris A, Dunn JA, et al. Science 2009; 323:1057-1060.

5 Panchanathan R, Duan X, Shen H, et al. J Immunol 2010; 185:73857393.

6 Theobald DL, Mitton-Fry RM, Wuttke DS. Anпu Rev Biophys Biomol Struct 2003; 32:115-133.

(Supplementary information is linked to the online version of the paper on the Cell Research website.) 